\title{
Modelling of sustainable development of megacities under limited resources
}

\author{
Sergey Sergeev ${ }^{1, *}$, Tatiana Kirillova ${ }^{1}$, and Irina Krasyuk $^{1}$ \\ ${ }^{1}$ Peter the Great St. Petersburg Polytechnic University, 29, Polytechnitcheskaya street, 195251, Russia
}

\begin{abstract}
The paper is devoted to topical issues of urban planning in relation to megacities. We consider this issue taking into account the limitations of natural, communal, and social resources. The problem is especially acute for the overwhelming majority of the state capitals, as well as cities with historical heritage that are of great interest due to the rapid development of tourism and the high mobility of the population in the world. The authors formalize the problem of determining the optimal ratio of the volume of urban buildings intended for non-residents to the total housing stock. We conduct economic calculations to determine the quantitative indicators that can be used as the basis for administrative measures aimed at limiting the flow of people arriving with the intention of temporarily deploying in megacities.
\end{abstract}

\section{Introduction}

Urban planning, as a rule, covers a significant time horizon. At the same time, a set of tasks related to practical functions is solved, where the arguments are economic indicators $[1,2]$, demography, ecology, and limited environmental resources intended for common use. Analysis of such indicators as the population size, the volume of housing construction carried out among the major cities of the world shows that the list of cities from Alpha to Alpha ++ coincides with the participants in the ranking of the most attractive places for tourists. For comparison, according to the GaWC (Globalization and World Cities Research Network), we present the Global city table as the most important nodes of the global economy, ranked by Alpha indicator in descending order and the List of the cities most visited by tourists ranked according to data provided by Euromonitor research company. Such megacities are characterized by the presence of an increased ratio of the volume of accommodation (hotels, hostels, tourist hostels, etc.) for non-residents to the total area of the housing stock. In addition, over the past few years, through the penetration of the concept of Web 3.0., the world's B-corporation networks have emerged, concentrating on online sites such as Airbnb, CouchSurfing, a powerful attraction potential for travelers. They take advantage of the integration with social networks such as facebook, as well as a wide range of mobile applications for smartphones on common platforms iOS and Android. At the same time, a global information and technology service field is implemented to simplify travel and accommodation in any city in the world. [3] In the number of persons

\footnotetext{
*Corresponding author: kirillova_tan@rambler.ru
} 
temporarily staying in the selected territory, it is also necessary to include the category of citizens migrating for the period of temporary earnings (Table 1).

Table 1. Comparison of data on global cities.

\begin{tabular}{|c|c|c|c|c|c|}
\hline NN & Global City & Attractiveness & 12 & Milan & Tokyo* \\
\hline 1 & New York* & Hong Kong* & 13 & Chicago & Prague \\
\hline 2 & London* & Bangkok & 14 & Mexico City* & Delhi \\
\hline 3 & Singapore & London* & 15 & Mumbai* & Vienna \\
\hline 4 & Hong Kong* & Singapore & 16 & Moscow* & Berlin \\
\hline 5 & Paris* & Pari** & 17 & Frankfort & Madrid \\
\hline 6 & Beijing & Dubai* & 18 & Madrid & Moscow* \\
\hline 7 & Tokyo* & New York* & 19 & Warsaw & Beijing \\
\hline 8 & Dubai* & Kuala Lumpur & 20 & Johannesburg & Athens \\
\hline 9 & Shanghai* & Rome & 21 & Toronto & Budapest \\
\hline 10 & Sydney & Taipei & 22 & Seoul* & Lisbon \\
\hline 11 & São Paulo & Seoul* & 23 & Saint Petersburg & Saint Petersburg \\
\hline
\end{tabular}

Thus, it becomes possible to formulate the research task. It is necessary to model [4] the use of the limited potential available to the city. At the same time, its composition can be both natural (environment, air, water) and technological (utilities, transport), as well as social (medicine, culture, law and order). As a result, it is necessary to find the maximum number of the total population, both permanently resident and non-residents, based on the potential limits of the loaded resources [5] of public use.

\section{Materials and Methods}

The solution of the problem will be sought using the methods of mathematical modeling [6], using the economic applications of game theory, a series of results of Queuing theory, and auxiliary formulas for describing stochastic processes from the economic point of view. We introduce a number of necessary formalisms, which make it possible to uniquely identify the parameters [7] of the investigated processes. We define common resource as $C R_{i}$. We set $i=1 \ldots \Omega$, where $\Omega-$ the amount of common resources concentrated in the territory under consideration. Considering transport, it is necessary to separate communications with external and internal environment. The category of transport that implements external communications includes railway, air communication, passenger port, radial roads. The second type is represented by urban traffic, metro, public transport. The next group $C R$ includes utilities such as sewage, electricity [8], water, fire, medical facilities, and garbage collection. The socially significant resources should also include natural, characterized by indicators of air pollution, water bodies, the presence of space in parks, the density of the population in the city. It is necessary to supplement these data $C R$ with the following specific types of resources considered in the mathematical model [9]. All the cities listed in Table 1 are characterized by a high concentration of tourist attractions, which are concentrated in a limited area. Such places of attraction are called POI (place of interest). Usually it is either a historical center of the city, which is typical for most European cities, or cultural objects (theaters, museums), or places of world-wide events (olympiads, military battles). We introduce the following notation: 
$\lambda_{i}$ - intensity of user flow $i-$ th $C R$, where $i=1 \ldots \Omega . \mu_{i}$ - capacity indicator $i-$ th $C R$ where $i=1 . . \Omega$.

Then, using Kendall's symbols for the model parameters in the form: $M / M / n / Q$ we can calculate the average time spent in finding and using $C R_{i}$ :

$$
\bar{T}=\bar{R}_{i} / \lambda_{i}
$$

where $\bar{R}_{i}=\frac{\rho_{i}^{2}\left(1-\left(M+1-M \rho_{i}\right) \rho_{i}^{M}\right)}{\left(1-\rho_{i}\right)\left(1-\rho_{i}{ }^{M+2}\right)}, \rho_{i}=\frac{\lambda_{i}}{\mu_{i}}, i=1 \ldots \Omega$,

$M$ - acceptable queue length for this resource. For this, we used the well-known solution of the Erlang equations. Now formally imagine the city as a set of resource users. For the issues considered in this problem, it is convenient to present the representation in a consolidated form, as a set of houses, hotels, hostels. We denote their number by $n$. Denote the load of each user as $l_{i}$ where $i=1 \ldots n$, then in vector form we can write down:

$$
\bar{\Lambda}=\left(l_{1}, l_{2}, \ldots, l_{n}\right)
$$

while the total load $L$ on the resource is: $L=\sum_{i=1}^{n} l_{i}$.

Next, we will establish the value of the weighted average variable costs $U$, and a function $g(l)$. The function reflects the value of the revenue per unit of the load of each user. We suppose that with the total load starting from a certain number $L>L_{0}$, the profitability of the resource falls both in connection with the activities of competitors, and because of the saturation of the market. This can be expressed by the relation:

$$
g^{\prime}(l)<0
$$

Further, for the same reasons, we add factors related to the negative component of the impact of the activities of these $n$ users on the resource and its natural limitations. It follows that the second derivative: $g^{\prime \prime}(l)<0$.

We write the ratio for calculating the $i-$ th user's winnings as:

$$
R_{i}=l_{i} g\left(l_{1}+l_{2}+\ldots+l_{n}\right)-U l_{i}=l_{i} g(L)-U l_{i}
$$

Then, assuming the existence of Nash equilibrium [10], there must exist a load value of the $i$ - th user $l_{i}^{*}$ under which the value of expression (3) reaches a maximum with the remaining components of the vector $(1): \bar{\Lambda}_{i}^{*}\left(l_{1}^{*}, l_{2}^{*}, \ldots, l_{i-1}^{*}, l_{i+1}^{*}, \ldots, l_{n}^{*}\right)$

We shall seek the solution of the problem by taking the partial derivatives $\frac{\partial R_{i}}{\partial l_{i}}$ from the extremum condition: $\frac{\partial R_{i}}{\partial l_{i}}=0$ for $i=1 \ldots n$. Further, denoting as $l_{-i}^{*}=\sum_{k \neq i} l_{k}^{*}$ we obtain the following expression: $g\left(l_{i}+l_{-i}^{*}\right)+l_{i} g^{\prime}\left(l_{i}+l_{-i}^{*}\right)-U=0$ for $i=1 \ldots n$.

Summing up the values at the desired equilibrium point $L^{*}$, we obtain the equation: 


$$
L^{*}=n \frac{U-g\left(L^{*}\right)}{g^{\prime}\left(L^{*}\right)}
$$

If we compare this expression with the search for a maximum from the point of view of the level $L_{0}$ - the optimal use of the resource by the city, then the extremum of the equation will be achieved if the following relation is satisfied: $L_{0}=\frac{U-g\left(L_{0}\right)}{g^{\prime}\left(L_{0}\right)}$. Taking into account (2) and the fact $n>1$, it's obvious that $L^{*}>L_{0}$. The meaning of the results you can see in calculations on real data.

\section{Results}

To illustrate the above procedure, we will calculate the above formulas using specific values obtained from the analysis of the municipal economy of St. Petersburg. From Russian cities, this is one of the most sought after points for travel of domestic and foreign tourists. This is confirmed by a survey of UNESCO and the WTO, according to which the northern capital is among the most attractive cities in the world in terms of its potential. Such a flow of visitors imposes a number of requirements on the organization of city services and provides a wide field for the development of optimal [11] solutions for the planning and implementation of all types of business associated with temporary stay in the city. In the economic theory, this problem has been developed insufficiently both in the general methodological aspect and in the field of practical implementation. To conduct analysis and formulate the commercial policy of the city administration on an extended horizon, it is necessary to apply well-developed mathematical methods and models [12]. When compiling them, it is necessary to take into account a number of factors:

- uneven demand for this type of services, not only related to annual seasonality, but also such periods as school holidays, traditions, public holidays;

- provide for the coordination of simulation results with GDS (Global Distribution System) systems, CRS (computer reservations system);

- business planning must comply with USALI (The Uniform System of Accounts for the Lodging Industry) standards;

- modeling should provide for the implementation of services not only for individual customers, but also wholesale consumers, as well as in the MICE (Meetings, Incentives, Conferences, Exhibitions) and TMC (Tourism Marketing Concepts) segments.

Let's consider separately each of the listed items. The study of issues related to the modeling of commercial processes should be carried out, on the one hand, by visual methods, which implies the use of simplified schemes. On the other hand, it is necessary to use the capabilities of computer technology and the application of tools of mathematics and statistics. As in any commercial segment, planning for the reception of visitors of the city is impossible without reliable estimates of future market needs. First of all, this is due to the fact that such an organization as, for example, a hotel, is closing in a long chain consisting of advertising, travel companies and intermediaries, each link being characterized by its own lag. Additionally, in such a business there is the influence of seasonality and, as already mentioned, there is still a correlation with socially significant events, vacations and other known market factors. Mathematics provides a number of ways to predict future values of a process based on extrapolation of known results. The simplest way is regression and trend analysis [13], but the polynomial trend realized in mass office products does not allow building analytical models. Next, it should be noted that the cycle for any type of 
service is not necessarily a one-off (maybe several seasons a year). Intervals between cycles within a year are also not necessarily the same, i.e. there is usually a non-uniform frequency characteristic of an asymmetric shape [14]. The initial data will be considered indicators of the number of arriving citizens for a fairly long period of time. It is desirable that it includes at least two complete annual cycles. For certainty, we will assume that the data is received monthly, which is consistent with the tax accounting. Using the possibilities of programming on a computer, one can obtain by simple means a function that describes the data on the number of visitors for the previous period. It is known that a periodic function can be represented as a Fourier series, then, adding it to a sufficient planning horizon, it is possible to continue calculations based on the data already obtained. Formally, it can be represented:

$$
F(x)=A_{0}+\sum_{k=1}^{+\infty} A_{k} \operatorname{Cos}\left(2 \pi k / T x+\theta_{k}\right),
$$

where $F(x)$ - function, to calculate the number of visitors, $A_{0}$ - constant characterizing the displacement, $A_{k}$ - amplitude of the k-th harmonic oscillation, $2 \pi k / T$ circular frequency of harmonic oscillation, $\theta_{k}$ - initial phase of the k-th oscillation. Our task is to determine the unknown coefficients of the Fourier expansion $A_{0}, A_{k}, \theta_{k}, T$. Since a non-linear model is needed for the solution, it is necessary to add the corresponding Solver for Nonlinear Programming [15]. In order to find a solution with data on the volume of guest flow by months, and the period can naturally be taken to be one year, then, for example, the expression for the first harmonic of the Fourier expansion looks as follows:

$$
F(m)=A_{0}+A_{1} \operatorname{Cos}\left(2 \pi \frac{1}{12}\left(m+\theta_{1}\right)\right)
$$

where $m$ - month number (tabular, not calendar). In addition, the target value and optimization criterion will be needed [16]. As a criterion, the minimum standard deviation of the calculated data is taken. The target value is also calculated using Solver for Nonlinear Programming. When choosing either DEPS Evolutionary Algorithm or SCO Evolutionary Algorithm, we proceed from the fact that DEPS (Differential Evolution and Particle Swarm Optimization) consists of two independent algorithms and represents each vector of the solution as coordinates in the dimension space of the arguments, simultaneously in each iteration choosing one of the two strategies and applying to the current solution vector. SCO (Social Cognitive Optimization) mimics the behavior of learning and sharing information. At each iteration searches for the solution, comparing it with the data already received. As a result, we already obtain in the analytical form a formalized expression for the intensity of resources. The coefficients $A_{0}, A_{k}, \theta_{k}, T$ will be used for further calculations. Using data on the tourist flow to St. Petersburg, the capacity of the POI, as a result of calculations on a computer, we obtain data on the intensity of the load, as well as a forecast for saturation of the city's capabilities as a visiting site from the condition of loss of attractiveness of its sights. 


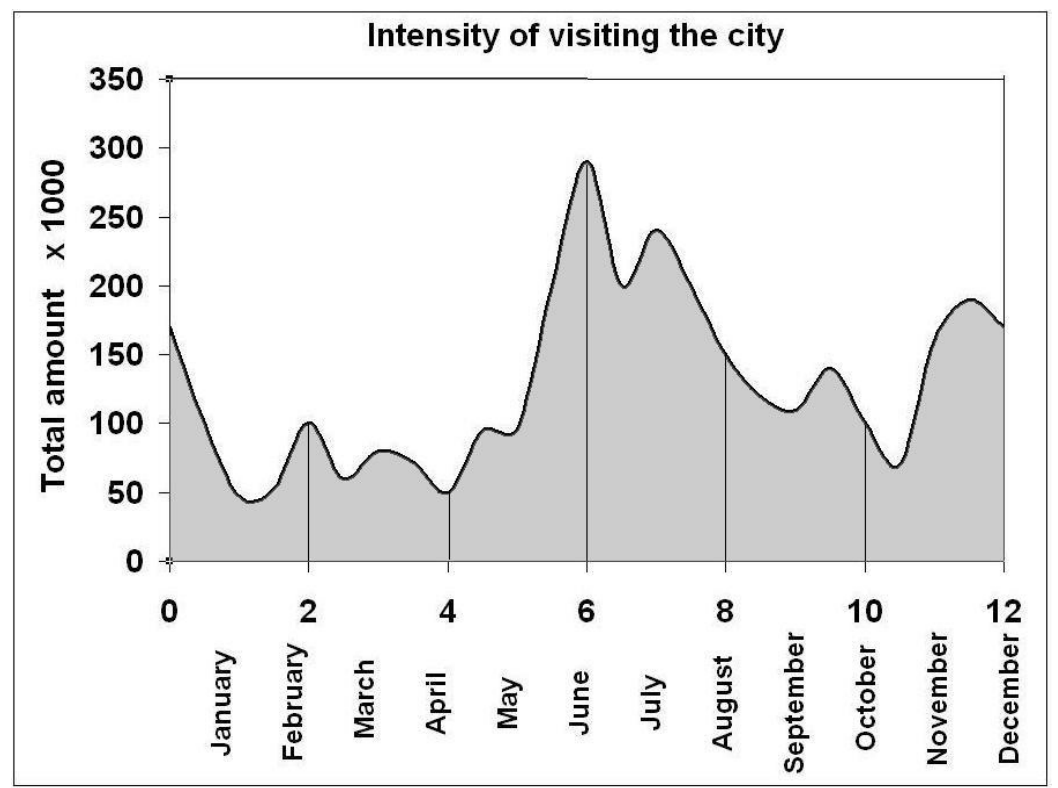

Fig. 1. Calculating the intensity of visiting the city.

Figure 2 shows data on the degree of satisfaction of requests. Its assessment is carried out as an indicator of how many POIs in reality managed to visit a visitor. The graph shows the percentage of the actual value of the POI from the planned one. Note that the data are averaged, for specific dates it is necessary to take into account the unevenness of the graph of Figure 1, with an inversely proportional dependence. Figure 2 also indicates the current level of ratio of visitors to the resident population.

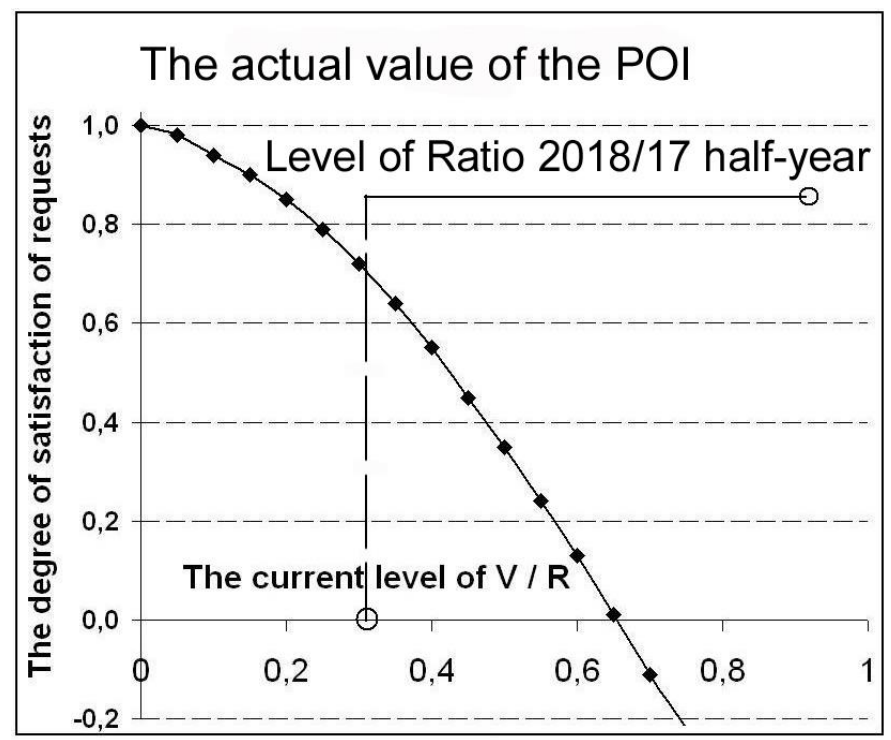

Fig 2. Level of satisfaction with requests.

The final result of the calculation should be a set of recommendations for the optimal reception of visitors. Turning to world experience, it can be concluded that the most common approach is to apply restrictive measures. It is known that there is usually no 
technical solution here, and it is within the competence of local authorities to establish rules of the game, administrative regulation. In this case, mathematical modeling serves to make recommendations based on the forecast for the adoption of these legal or other measures that will be made during the next phase of business in the hospitality industry by constraints formalized in the compilation of dynamic equations.

\section{Discussion}

In Table 1, an asterisk indicates cities in which, according to the information of GaWC, there are a maximum number of foreigners, the need to increase the share of non-resident housing stock, the most intense passenger traffic by main types of transport and the largest population. At the same time, their authorities were the first to introduce restrictions. There were many interrelated reasons for their initiation. The restriction was introduced to tourists visiting historical sites in order to prevent their destruction, as well as restrict access to a number of unique natural sites. Another serious limitation was the lack of hotels in demanded seasonal visit periods. Another circumstance was transport problems, traffic jams, and a decrease in the average speed of traffic. The listed cities are at the same time the most important transport hubs; a similar capacity limit is experienced by seaports due to cruise ships and air harbors. One of the most tangible results was a sharp increase in air pollution in these densely populated cities. But the most significant reason for the authorities is the discontent of resident residents, the creation of hotbeds of social tension due to the limited resource of public order services. As a result, the paradigm for improving the quality of life of visitors was formulated. An effective solution was the introduction of an environmental tax on visitors. Its size is differentiated depending on the level of the hotel. In order to exclude unaccounted visitors, there are large penalties for using resources such as Airbnb, CouchSurfing. In addition, the city authorities impose restrictions on the construction of new hotels, located in separate buildings. As an alternative, we propose to place hotels in high-rise buildings with a developed infrastructure. Figure 3 shows the calculation of the optimal (averaged) Ecotax. The graph represents the calculations as theoretical, from the formulas based on the model considered in this paper, and obtained from real data.

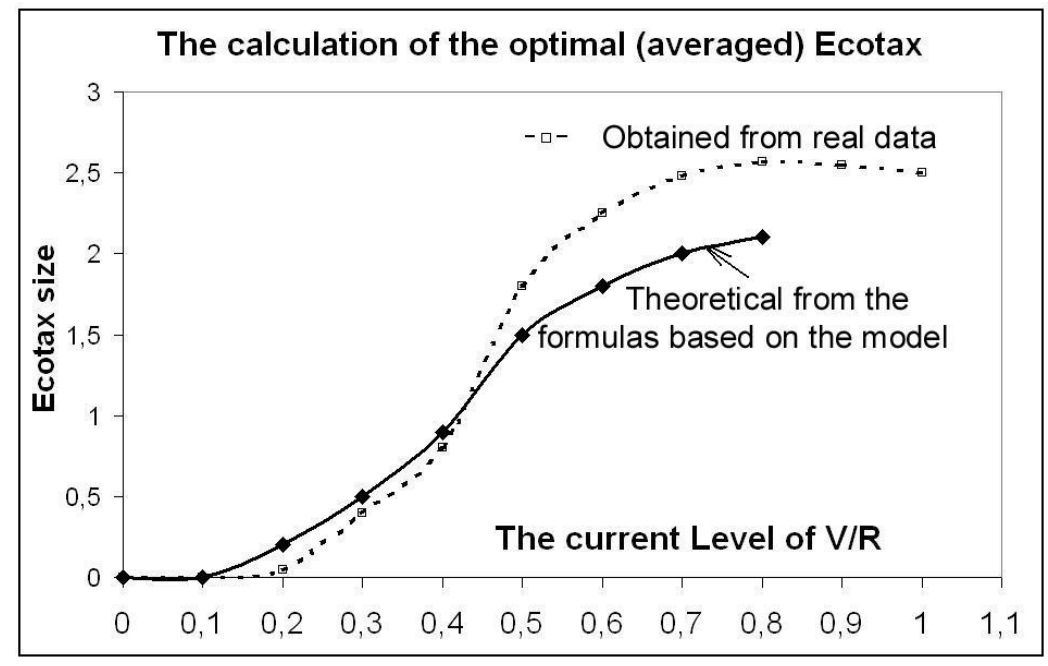

Fig. 3. Calculation of the optimal value Ecotax. 
Note that the theoretical calculation covers only part of the possible ratio of the number of visitors to residents. On the contrary, real data show strongly different character at large values of this ratio. This is explained by the reasons that can't be taken into account in the model and are of a social nature. First of all, this refers to the regions in which business on tourists is the main share of the economy. For their populations, a maximum influx of visitors is required during the high season, since the rest of the year most residents will not have other earnings.

\section{Conclusions}

In the conduct of economic activity, most often by competing business entities in the same territory, over time, factors related to the sphere of human values appear. Since hotels in attractive cities are concentrated around historic centers with attractions, the problem of using this resource is very acute in Madrid, Barcelona, Rome, Venice, Tallinn, St. Petersburg, and dozens of other landmark places for travelers. Theoretical and methodological provisions for the formation of a forecasting system that interacts with the current and prospective management of hotel enterprises in a single complex that determines the target orientation and ensures the reality of achieving the necessary economic and social results in the development of hotels have not been adequately developed in the economic literature. From the standpoint of the business analyst, who forms theoretical models, multidirectional processes must be taken into account in the problem under consideration. This is an increase in the burden on resources and the resulting decline in the quality of these types of common resources, while simultaneously assessing the negative component of the impact of business processes. Various socioeconomic factors necessitate the search for various measures to address the issues of regulating the flow of tourists in megacities. 1. Administrative regulation of this business segment is necessary. Many cities are already solving this problem. One of the ways to solve the problems is to develop mechanisms for regulating the volumes of non-resident housing stock. One such mechanism is the restriction in the construction of new buildings for hotels and hotel accommodation in high-rise buildings. At the same time, it is necessary to take into account a number of restrictions on high-rise construction of non-resident housing stock in megacities. Another mechanism is the introduction of an environmental fee or tourist tax. This process is everywhere, in many cities and countries, and the figures vary significantly, but the general principle remains the same - the amount of tax should balance between the decrease in the number of tourists and the damage from the increase in the intensity of the flow of guests. The methods outlined in this paper make it possible to scientifically justify the dynamics of these multidirectional processes and simulate the main parameters of such interaction on the planning horizon in 2-3 years. 2. The usual static modeling and stationary solution for forecasting is not enough. It is necessary to use computer calculations on dynamic models with the use of scientific methods based on serious mathematical methods.

\section{References}

1. O. Iliashenko, S. Krasnov, S. Sergeev, E3S Web of Conferences 33, 030062017 (2018)

2. I.A. Krasyuk, Y.Y. Medvedeva, IEEE Conference on Data Science, Challenges of Digital Transformation (IEEE DSDT 2018), 482-488 (2018)

3. S.V. Krasnov, S.M. Sergeev, N.V. Mukhanova, A.N. Grushkin, 2017 6th Int. Conf. on Reliability, Infocom Technologies and Optimization, Trends and Future Directions, ICRITO 2017, 553-558 (2017) 
4. O.V. Grishchenko, V.S. Kireev, L.I. Dubrova, M.B. Yanenko, R.Y. Vakulenko, Int. Journal of Economics and Financial Issues 6(8), 166 -172 (2016)

5. I.V. Kapustina, T.V. Kirillova, O.V. Ilyina, O.A. Razzhivin, P.A. Smelov, Int. Journal of Applied Business and Economic Research 15(11), 1-10 (2017)

6. L.N. Borisoglebskaya, S.M. Sergeev, Journal of Applied Economic Sciences 2018 XII8(54), 2423-2448 (2017)

7. A. Chernikova, S. Golovkina, S. Kuzmina, T. Demenchenok, IOP Conference Series: Earth and Environmental Science, 90(1) (2017)

8. M.B. Yanenko, International Business Management Year 10(26), 5991-5995 (2016)

9. Krymov S.M., Kapustin I.V., M.V. Colgan, XVI all-Russian scientific-practical conference "Planning and providing training for industrial and economic complex of region", 130-133 (2017)

10. E.M. Kiseleva, E.I. Artemova, I.L. Litvinenko, T.V. Kirillova, V.A. Tupchienko, W. Bing, Int. Journal of Applied Business and Economic Research 15(13), 231-242 (2017)

11. V.V. Provotorov, Constructive Nonsmooth Analysis and Related Topics (Dedicated to the Memory of V.F. Demyanov), CNSA 2017, 7974008 (2017)

12. V.V. Provotorov, International Conference on "Stability and Control Processes in Memory of V.I. Zubov, SCP", 126-128 (2015)

13. E. Korchagina, P. Shilo, Financial Environment and Business Development. Proc. of the 16th Eurasia Business and Economics Society Conference, Springer Int.Publishing 4, 315-323 (2017)

14. A. Aleksandrov, E. Aleksandrova, A. Zhabko, Circuits, Systems, and Signal Processing 35(10), 3531-3554 (2016)

15. I.A. Krasyuk, V.V. Bakharev, N. A. Kozlova, D.D. Mirzoeva, XVI all-Russian scientific-practical conference "Planning and providing training for industrial and economic complex of region", 52-54 (2017)

16. V.V. Provotorov, V.I. Ryazhskikh, Yu.A. Gnilitskaya, Vestnik Sankt-Peterburgskogo Universiteta, Prikladnaya Matematika, Informatika, Protsessy Upravleniya 13(3), 264277 (2017) 